\title{
An Investigation of Flumazenil to Antagonize Gamma-Hydroxybutyrate Intoxication in a Murine Model
}

\author{
David C. Lee, MDab, Wayne A. Satz, MD ac, Timothy Dougherty, MD', Tucker Greene, $M D^{\mathrm{a}}$ \\ a Department of Emergency Medicine, Drexel University/Medical College of Pennsylvania \\ b Department of Emergency Medicine, North Shore University Hospital \\ c Department of Emergency Medicine, Temple University Hospital
}

\begin{abstract}
Objective: Prior animal studies have suggested that flumazenil may blunt GHB's sedative-hypnotic affects. We hypothesized that flumazenil would decrease the affects of GHB in a murine model of intoxication.

Methods: We performed a controlled, pilot experiment using 32 mice divided into 3 groups. All mice received intraperitoneal injections of GHB $(1.5 \mathrm{~g} / \mathrm{kg})$. Group I received sham injections at time 0 , and then GHB at 5 minutes. Group II received flumazenil $(0.3 \mathrm{mg} / \mathrm{kg})$ at time 0 , and then GHB at 5 minutes. Group III received sham injection at time 0 , then GHB at 5 minutes, and then 4 escalating flumazenil doses administered at 3-minute intervals $(0.003$ to $1 \mathrm{mg} / \mathrm{kg})$. We measured certain functions: time to loss/recovery of righting reflexes (RR), time to sprawl/recovery of sprawl (postural tone [PT]), and death.

Results: There were statistically significant delays in the loss of PT and shortened recovery time to RR in pre-treated mice (group II) versus controls (group I). There were no differences in group III versus group I for any outcome parameters.

Conclusions: In this model, pre-dosing flumazenil prior to GHB administration delayed clinical intoxication.
\end{abstract}

\section{INTRODUCTION}

Gamma-hydroxybutyrate (GHB) is a sedative-hypnotic agent that has been increasing in popularity with recreational illicit drug users. Patients who overdose with GHB often present with sedation, obtundation, and respiratory depression [1]. Clinical management of patients with GHB intoxication predominantly consists of supportive care. Prior animal studies have suggested that flumazenil, a competitive benzodiazepine receptor antagonist, may blunt the affects of GHB [2-5]. Schmidt-Mutter and colleagues reported that flumazenil blunted the anxiolytic affect of GHB in rats [5]. However, Carai and colleagues recently reported that flumazenil did not alter mortality rates in mice given a toxic dose of GHB4. There are no reported human studies describing the use and clinical effect of flumazenil in GHB overdoses.

The exact mechanism of GHB toxicity is unclear. Since GHB is a short chain fatty acid that structurally resembles gammaaminobutyric acid (GABA), several authors have suggested that GHB may interact with GABA A receptors, GABA B receptors, independent GHB receptors, or a combination of these $[2,4,6]$. We sought to describe the clinical interactions of GHB and flumazenil in a murine model of significant GHB intoxication. We hypothesized that flumazenil would decrease the sedative effects of GHB in a murine model of significant GHB intoxication. In this pilot study, we did not calculate initial power samples.

Keywords: gamma-hydroxy butyrate, flumazenil, poisoning, mice

Note: This study was presented at the Society of Academic Medicine Scientific Meeting in May of 1997 in Washington, DC. Corresponding Author: David Lee, MD, Department of Emergency Medicine, North Shore University Hospital, 300 Community Drive, Manhasset, NY 11030. Email: dlee@nshs.edu 


\section{METHODS}

\section{Study Design}

We performed a controlled, interventional, pilot laboratory experiment using mice. The study protocol was approved by our Animal Care and Utilization Committee and complied with the National Institute of Health's guidelines for ethical animal research.

\section{Study Protocol}

We obtained 32 conditioned Swiss Webster mice with an average weight of 25 grams. The mice were divided into 3 groups. All 3 groups received $1.5 \mathrm{~g} / \mathrm{kg}$ of $\mathrm{GHB}$ by intraperitoneal (IP) injection. We chose this dose of GHB since this has been roughly reported as the median lethal dose (LD50) [2]. Group I $(n=8)$ received sham IP injections of normal saline at time 0 , and then GHB at 5 minutes. Group II $(n=12)$ received $0.3 \mathrm{mg} / \mathrm{kg}$ of flumazenil at time 0 , and then GHB in 5 minutes. Group III $(n=12)$ received sham injection of normal saline at time 0 , then GHB at 5 minutes, and then 4 escalating IP doses of flumazenil every 3 minutes $(0.003 \mathrm{mg} / \mathrm{kg}, 0.03 \mathrm{mg} / \mathrm{kg}$, $0.3 \mathrm{mg} / \mathrm{kg}, 1 \mathrm{mg} / \mathrm{kg}$ ). The following parameters were measured: time to loss and recovery of righting reflexes, time to sprawl and recovery of sprawl (postural tone), and death. Time measurements began at the point of GHB injection. Investigators, who were blinded to the treatment group, continually observed and evaluated the study animals. Subjects were stimulated and tested if it was unclear of the status of postural tone and righting reflex.

\section{OUTCOME MEASURES}

Time intervals were recorded starting from the injection of GHB until loss of postural tone and righting reflex. Time intervals were recorded starting from GHB injection to the recovery of postural tone and righting reflex. Mortality was documented and all remaining animals were sacrificed at 4 hours.

\section{DATA ANALYSIS}

We compared the mean times of various intervals of the 3 groups, and parametric data were analyzed with Analysis of Variance with Tukey post-hoc tests. Frequency data were analyzed with Fisher's Exact test. $P<0.05$ was considered statistically significant.

\section{RESULTS}

Mean times are summarized in Table 1. Compared to the control group (group I), mice pre-treated with flumazenil (group II) showed a statistically significant delay in the loss of postural tone and shortened recovery time to righting reflex. Compared to the control group (group I), animals in the post-treatment flumazenil group (group III) did not show significant differences in outcomes. Furthermore, one animal in group I died and all other animals in group I recovered within 4 hours. No animals in groups II or III died.

Table 1 - Mean times (minutes) with standard deviations (SD)

\begin{tabular}{|c|c|c|c|c|c|c|}
\hline & Loss of PT & Loss of RR & Recovery of RR & Recovery of PT & Total-PT & Total-RR \\
\hline Group I & 4.8 SD 0.3 & 20.5 SD9.6 & 101 SD 24.6 & 153 SD 10.0 & 149 SD 28.3 & 80 SD 17.0 \\
\hline \multicolumn{7}{|l|}{$\begin{array}{l}\text { Control } \\
(n=8)\end{array}$} \\
\hline Group II & 9.8 SD $2.8^{\star}$ & 20.5 SD 4.5 & 30 SD $24.3^{*}$ & 143 SD 10.4 & 132 SD 10.4 & 45 SD $24.3^{*}$ \\
\hline $\begin{array}{l}\text { Flu-GHB } \\
(n=12)\end{array}$ & $(p=0.05)$ & & $(p=0.027)$ & & $(p=0.029)$ & \\
\hline Group III & 5.8 SD 1.0 & 20.9 SD 8.0 & 118 SD 24.3 & 151 SD 20.8 & 145 SD 20.8 & 96 SD 24.3 \\
\hline \multicolumn{7}{|l|}{$\begin{array}{l}\text { GHB-FLU } \\
(n=12)\end{array}$} \\
\hline \multicolumn{7}{|c|}{$P T=$ postural tone } \\
\hline \multicolumn{7}{|c|}{ Loss of PT is the time from GHB injection to the first recorded loss of postural tone } \\
\hline \multicolumn{7}{|c|}{ Loss of RR is the time from GHB injection to the first recorded loss of righting reflex } \\
\hline \multicolumn{7}{|c|}{ Recovery of RR is the time from GHB injection to the first recorded recovery of righting reflex } \\
\hline \multicolumn{7}{|c|}{ Recovery of PT is the time from GHB injection to the first recorded recovery of postural tone } \\
\hline \multicolumn{7}{|c|}{ Total-PT is the time interval from the loss of postural tone to the recovery of postural tone } \\
\hline \multicolumn{7}{|c|}{ Total-RR is the time interval from the loss of righting reflex to the recovery of righting reflex } \\
\hline
\end{tabular}




\section{LIMITATIONS}

This was a pilot study with a limited number of subjects. We did not establish a LD50 intraperitoneal dose of GHB in our study animals but used a previously published dosing regimen [3]. Although mice were profoundly sedated after receiving a reported LD50 dose of GHB, only 1 subject died during the experiment. We are unclear as to why our subjects experienced a low mortality and whether handling of the animals provided sufficient tactile stimulation to prevent death. We attempted to measure righting reflex and postural tone with minimal intervention. Righting reflex and postural tone are study tools used to evaluate basic instinctive murine function. Righting reflex occurs when a mouse is placed on its side and the mouse will instinctively set itself upright on all four legs. Sprawl or postural tone is evaluated by observing the mouse standing upright on all four legs. Loss of postural tone occurs when the mouse slumps onto its abdomen. Furthermore, we are unclear if subjects were given a larger dose of $\mathrm{GHB}$; there may have been a greater clinical effect of flumazenil.

\section{DISCUSSION}

Prior studies have investigated the interaction between nonlethal doses of GHB and flumazenil [2,5-7]. The literature describing the interactions between toxic doses of GHB and flumazenil is scant. Carai and colleagues recently reported that flumazenil did not have any affect on mice given large doses of GHB $(7 \mathrm{~g} / \mathrm{kg})$ [4]. All mice given the aforementioned dose expired within 24 hours. Compared to Carai's study, we used lower doses of GHB (up to $1 \mathrm{mg} / \mathrm{kg}$ ) and lower doses of flumazenil. In our study, the mice in group II that received pre-treatment with flumazenil had a delay of the sedating effects of GHB. Since flumazenil is not known to interact with GHB or GABA B receptors, this suggests that some of GHB's effect may be through the benzodiazepine-GABA A receptor complex suggested by SchmidtMutter and colleagues. In their murine experiment, SchmidtMutter and colleagues used lower doses of GHB $(250 \mathrm{mg} / \mathrm{kg})$ and higher doses of flumazenil $(10 \mathrm{mg} / \mathrm{kg})$ in mice and reported that flumazenil blunted the anxiolytic effects of GHB [5].

We decided to use lower doses of flumazenil than Carai or Schmidt-Mutter because we suspected that high doses of flumazenil might act as an agonist at the benzodiazepine receptor, facilitating the triggering of the GABA A receptors. Adachi and colleagues, in their study investigating the interaction between flumazenil and propofol, reported that high doses of flumazenil increased the sedative-hypnotic effects of propofol $[8,9]$. In Carai's study, the use of high doses of flumazenil may have potentiated the sedative properties of GHB [4].

\section{CONCLUSION}

Pre-dosing flumazenil prior to GHB administration significantly delayed clinical GHB intoxication. There were no statistically significant differences with outcome measures using high doses of flumazenil administered after GHB. In this small pilot study using a murine model, our preliminary results do not support the routine clinical use of flumazenil in patients with GHB obtundation.

The authors have no potential financial conflicts of interest to report.

\section{REFERENCES}

1. Chin RL, Sporer KA, Cullison B, Dyer JE, Wu TD. Clinical course of gamma-hydroxybutyrate overdose [see comments]. Ann Emerg Med 1998; 31:716-22.

2. Gerra G, Caccavari R, Fontanesi B, Marcato A, Fertonani AG, Maestri D, et al. Flumazenil effects on growth hormone response to gamma hydroxybutyric acid. Int Clin Psychopharmacol 1994; 9:211-5.

3. Mason PE, Kerns WP, 2nd. Gamma hydroxybutyric acid (GHB) intoxication. Acad Emerg Med 2002; 9:730-9.

4. Carai MA, Colombo G, Gessa GL. Resuscitative effect of a gamma aminobutyric acid $\mathrm{B}$ receptor antagonist on gammahydroxybutyric acid mortality in mice. Ann Emerg Med 2005; 45:614-9.

5. Schmidt-Mutter C, Pain L, Sandner G, Gobaille S, Maitre M. The anxiolytic effect of gamma-hydroxybutyrate in the elevated plus maze is reversed by the benzodiazepine receptor antagonist, flumazenil. Eur J Pharmacol 1998; 342:21-7.

6. McMahon LR, Coop A, France CP, Winger G, Woolverton WL. Evaluation of the reinforcing and discriminative stimulus effects of 1,4 butanediol and gamma-butyrolactone in rhesus monkeys. Eur J Pharmacol 2003; 466:113-20.

7. Gerra G, Caccavari R, Fontanesi B, Fertonani AG, Maestri $\mathrm{D}$, Avanzini $\mathrm{P}$, et al. Naloxone and metergoline effects on growth hormone response to gamma- hydroxybutyric acid. Int Clin Psychopharmacol 1995; 10:245-50.

8. Adachi YU, Watanabe K, Higuchi H, Satoh T. Flumazenil reduces the hypnotic dose of propofol in male patients under spinal anesthesia. J Anesth 2002; 16:9-12.

9. Adachi YU, Watanabe K, Higuchi H, Satoh T. High-dose flumazenil potentiates the hypnotic activity of propofol, but not that of thiopental, in ddY mice. Acta Anaesthesiol Scand 2001; 45:848-52. 